\title{
Predictive value of VEGF gene polymorphisms for metastatic colorectal cancer patients receiving first-line treatment including fluorouracil, irinotecan, and bevacizumab
}

\author{
Vincenzo Formica • Raffaele Palmirotta - Girolamo Del Monte • Annalisa Savonarola • \\ Giorgia Ludovici • Maria Laura De Marchis • Italia Grenga • Michele Schirru • \\ Fiorella Guadagni • Mario Roselli
}

Accepted: 3 December 2010 / Published online: 29 December 2010

(C) Springer-Verlag 2010

\begin{abstract}
Purpose The aim of this study is to evaluate the influence of germline vascular endothelial growth factor (VEGF) gene polymorphisms (VGPs) on the efficacy of the antiVEGF antibody bevacizumab (Bev) in metastatic colorectal cancer (MCRC) patients.

Methods Forty MCRC patients eligible for a first-line therapy were enrolled in this prospective trial and treated with FOLinate/Fluorouracil/Irinotecan (FOLFIRI) + Bev $($ male/female $=22: 18$, age $($ median $)=61$ years $)$. Eight VGPs within the promoter/5'UTR region were evaluated in patient blood samples. Primary endpoint was association between VGPs and median progression-free survival (mPFS). Overall radiological response rate (ORR), overall
\end{abstract}

V. Formica $(\bowtie) \cdot$ I. Grenga $\cdot$ M. Schirru $\cdot$ M. Roselli

Medical Oncology Unit, Internal Medicine Department,

'Tor Vergata' Clinical Center University of Rome,

Viale Oxford,

0133 Rome, Italy

e-mail: v.formica@fastwebnet.it

V. Formica $\cdot$ M. Roselli

IRCCS San Raffaele,

Via della Pisana 235,

00163 Rome, Italy

R. Palmirotta • A. Savonarola · G. Ludovici · M. L. De Marchis •

F. Guadagni

Department of Laboratory Medicine and Advanced

Biotechnologies, IRCCS San Raffaele,

Via della Pisana 235,

00163 Rome, Italy

G. Del Monte

San Raffaele Rocca di Papa Clinical Center,

via Ariccia 16-00040, Rocca di Papa,

Rome, Italy survival (OS), and toxicity were assessed as secondary outcomes.

Results VGPs $-2578,-1512,-1451,-1411$, and -460 were in complete linkage disequilibrium and therefore analyzed as haplotype (two variants: Haplo1: A-18 bp insertion-T-4G-C and Haplo2: C-18 bp deletion-C-5G-T, respectively). Seventeen patients Haplo2/Haplo2 had significantly shorter mPFS compared to 23 patients Haplo1/ Haplo1 or Haplo1/Haplo2 (mPFS, 9 vs. 15.4 months, respectively, $p=0.02$; hazard ratio (HR), 2.64). Also, VGPs $-152(\mathrm{G} / \mathrm{G}$ vs. G/A+A/A) and -1154 (G/G vs. G/ $\mathrm{A}+\mathrm{A} / \mathrm{A}$ ) were significantly associated with PFS (mPFS, 8.9 vs. 15.4 months, $p=0.007$; HR, 3.53 and 9.8 vs. 16 months, $p=0.03$, HR, 2.32, respectively). In the multivariate analysis including also biochemical variables known to influence prognosis, VGP -1154 retained an independent predictive value for $\mathrm{mPFS}(\mathrm{G} / \mathrm{G}$ over $\mathrm{G} / \mathrm{A}+\mathrm{A} / \mathrm{A}=$ $\mathrm{HR}, 4.43 ; p=0.02$ ). With regard to ORR, only VGP -634 was significantly associated with response $(\mathrm{G} / \mathrm{G}$ vs. $\mathrm{G} / \mathrm{C}+\mathrm{C} / \mathrm{C}=$ $64 \%$ vs. $14 \%, p=0.03$ ). No significant influence on OS and toxicity by the investigated VGPs was observed.

Conclusions Although these data need to be confirmed in larger trials, investigation of germline VGPs may help identify patients who are more sensitive to anti-VEGF agents.

Keywords Bevacizumab - VEGF gene polymorphisms . Metastatic colorectal cancer patients $\cdot$ Predictive markers

\section{Introduction}

Inoperable metastatic colorectal cancer (MCRC) is an incurable disease that would affect approximately $20 \%$ of 
the nearly 150,000 new colorectal cancer cases expected to be diagnosed in 2009 in the United States [1].

Monoclonal antibodies (i.e., bevacizumab, cetuximab, and panitumumab) have significantly improved overall survival (OS) of MCRC patients, with median OS (mOS) of patients receiving these agents being nearly twice as long as it was 10 years ago: $20-24$ months vs. 1012 months [2].

Bevacizumab is a recombinant, humanized monoclonal antibody against the vascular endothelial growth factor (VEGF). It has proven antiangiogenic and vasculonormalizing properties [3] and has European and US regulatory approval for the treatment of MCRC patients in combination with fluoropyrimidine-based chemotherapy (www.emea.europa.eu, www.fda.gov).

Unlike other targeted therapies $[4,5]$, no clinical or biological factors clearly predictive of bevacizumab efficacy have as yet been identified [6].

VEGF plays a pivotal role in cancer neoangiogenesis and its importance in colorectal cancer growth and development has been extensively documented. It is secreted by both tumor and normal cells during cancer processes. VEGF originating from normal myeloid cells in response to tumor stimulation may be essential in tumorigenesis and may reduce susceptibility of tumors to standard chemotherapy [7].

Germline polymorphisms of the VEGF gene influencing VEGF expression in normal cells may therefore have an impact on tumorigenesis, tumor progression, and response to anti-VEGF agents [8].

Previous studies have demonstrated that the presence of VEGF single nucleotide polymorphisms (SNPs) in the promoter and 5' untranslated region (UTR) regions may be a risk factor for prostate and bladder cancer development $[9,10]$. Moreover, VEGF SNPs may have a prognostic value for non-small cell lung, colorectal, ovarian, and breast cancer patients [11-14].

The influence of VEGF SNPs on the outcome of a bevacizumab-treated patient has been previously investigated for breast and ovarian cancer $[15,16]$. The aim of the present study was to explore the effect of a number of VEGF gene polymorphisms (VGPs) on bevacizimab efficacy and toxicity in MCRC patients.

\section{Patients and methods}

Patients with histologically confirmed diagnosis of colorectal cancer and measurable metastatic disease at standard CT scan were eligible for this prospective observational study.

They were required to be chemotherapy-naïve for metastatic disease. Adjuvant treatment was allowed provided it was completed six or more months prior to study entry.

Patients had to have ECOG performance status $0-1$ and adequate hematological, hepatic, and renal functions. Prior treatment with monoclonal antibodies was not allowed.

A standard first-line chemotherapy regimen containing fluorouracil-leucovorin-irinotecan (FOLFIRI) was defined to be administered in association with bevacizumab.

Even though published data have not shown a clear difference between irinotecan- and oxaliplatin-based treatment in combination with bevacizumab $[17,18]$, the chosen regimen was FOLFIRI as oxaliplatin might have been administered before study entry as a part of adjuvant therapy.

All patients were therefore treated with the following drug doses and schedules: FOLFIRI+bevacizumab: irinotecan $180 \mathrm{mg} / \mathrm{m}^{2} /$ day 1 , bolus 5 -fluorouracil $400 \mathrm{mg} / \mathrm{m}^{2}$ days 1 and 2 , folinic acid $200 \mathrm{mg} / \mathrm{m}^{2}$ days 1 and 2 , continuous infusion of 5-fluorouracil $1,200 \mathrm{mg} / \mathrm{m}^{2}$ for $48 \mathrm{~h}$ starting from day 1, bevacizumab $5 \mathrm{mg} / \mathrm{kg}$ day 2; cycles were repeated every 2 weeks.

Exclusion criteria for study entry were: coagulation disorders, clinically relevant cardiovascular disease, or other cancers within the previous 5 years, except for adequately treated squamous or basal cell carcinoma of the skin or carcinoma in situ of the cervix.

Blood sample for VEGF gene genotyping was collected for all patients on day 1 of the first cycle, before treatment administration.

Treatment was continued until tumor progression, unacceptable toxicity, or request of termination by individual patients.

Clinical and biochemical evaluation of toxicity was performed before each cycle and graded according to the NIH Common Terminology Criteria for Adverse Events version 3.0 (http://ctep.cancer.gov/protocolDevelopment/ electronic_applications/ctc.htm).

Assessment of response was evaluated after every six cycles of treatment by comparison of a thorax/abdomen/ pelvis CT scan, according to RECIST criteria [19], with the baseline $\mathrm{CT}$ scan that had been performed within 45 days prior to chemotherapy initiation.

Data on gender, age, ethnicity, number of organs involved by metastatic disease, ECOG performance status (PS), histological features, circulating tumor markers (carcinoembryonic antigen (CEA) and carbohydrate antigen 19.9 (CA 19.9)), routine hematology, and biochemical blood tests were collected before treatment commencement. ECOG PS evaluation, CEA, CA 19.9, routine hematology, and biochemical blood tests were also performed during treatment at each cycle. Demographic, clinical and laboratory data were analyzed as potential predictors of patient outcome. Baseline data were considered evaluable if 
collected within 2 weeks before the start of the treatment. Metastatic sites of disease were grouped into five categories (liver, lymph nodes, peritoneum, lungs, and other). CEA and CA19.9 were performed in a single laboratory with the laboratory-defined upper limit of normal being $5 \mathrm{ng} / \mathrm{mL}$ and $35 \mathrm{UI} / \mathrm{mL}$, respectively. Biochemical variables known to have prognostic value in MCRC were evaluated for their association with patient outcome $[20,21]$ by using a Cox regression multivariate analysis (see below).

Since the present study focused mainly on laboratory predictors of outcome in order to decrease inter-observer variability, we did not include in the multivariate analysis clinical factors (e.g., performance status, etc.).

All patients signed an informed consent to blood collection for genotyping and treatment according to the study protocol.

The study was approved by the local ethics committee and Institutional Review Board and therefore performed in accordance with the ethical standards laid down in the 1964 Declaration of Helsinki.

\section{Molecular analysis of VEGF polymorphism}

All blood samples for genotyping were stored at $-80^{\circ} \mathrm{C}$ within $6 \mathrm{~h}$ of collection. Samples were then processed all at once approximately 6 months after the last patient was enrolled with outcome results being blinded for the investigators performing the genotype analysis. DNA was isolated from whole blood using the MagNA Pure LC instrument (Roche Diagnostics, Germany) and the MagNA Pure LC total DNA isolation kit I (Roche Diagnostics) according to the manufacturer's instructions.
VGPs to be tested were selected from the promoter/ 5'UTR (OMIM \# 192240) according to published data of the literature showing the highest polymorphic rates.

The region upstream of the start codon (i.e., the promoter/ 5 'UTR) has been shown to contain all the major regulatory VEGF gene sites [22]. In particular, the VEGF -2578 CC and -1154 GG genotypes have been associated with a higher VEGF expression [23].

Gene regions of interest were amplified using three pairs of primers spanning from $\mathrm{nt}-2713$ to $\mathrm{nt}-585$ (Table 1). Nucleotides position was conventionally numbered from the major transcription site [24-26] based on the sequence of the VEGF gene (Genebank Accession Number AF022375 and Ensembl Accession Number ENSG00000112715).

Standard polymerase chain reaction (PCR) was performed in a Veriti 96 Well Thermal Cycler (Applied Biosystems, CA) using HotStarTaq Master Mix (HotStarTaq Master Mix Kit, QIAGEN Inc.). In order to exclude pre-analytical and analytical errors, all sequencing analyses were carried out on both DNA strands using Big Dye Terminator v3.1 Cycle Sequencing kits (Applied Biosystems), run on an ABI 3130 Genetic Analyzer (Applied Biosystems) and repeated on PCR products obtained from new nucleic acid extractions.

\section{Statistical analysis}

The primary endpoint was to evaluate the predictive value of selected VGPs for progression-free survival (PFS), defined as the period beginning on the first day of treatment and ending with the first observation of disease

Table 1 Investigated VEGF gene polymorphisms (VGPs), numbered from the major transcription site, with respective http://www.ncbi.nlm.nih. gov/projects/SNP/ accession numbers, polymerase chain reaction (PCR) primer pairs, product size, and reaction conditions

\begin{tabular}{|c|c|c|c|c|c|}
\hline VGP & $\begin{array}{l}\text { Sequence } \\
\text { polymorphisms }\end{array}$ & Primers & $\begin{array}{l}\text { Gene } \\
\text { region }\end{array}$ & $\begin{array}{l}\text { PCR product } \\
\text { size (bp) }\end{array}$ & $\begin{array}{l}\text { PCR annealing } \\
\text { temp }\left({ }^{\circ} \mathrm{C}\right)\end{array}$ \\
\hline $\begin{array}{l}\text { rs699947 } \\
\text { rs35569394 }\end{array}$ & $\begin{array}{l}-2578 \mathrm{~A} \rightarrow \mathrm{C}^{\mathrm{b}} \\
-151218 \mathrm{bp} \\
\text { Insertion } \rightarrow \text { Deletion }\end{array}$ & \multirow[t]{3}{*}{$\begin{array}{l}\text { F5'-TGA GGA TGG GGC TGA CTA GGT AAG-3' } \\
\text { R5'-TGC GTG ATG ATT CAA ACC TAC CCG-3' }\end{array}$} & \multirow[t]{3}{*}{ Promoter } & \multirow[t]{3}{*}{567} & \multirow[t]{3}{*}{65} \\
\hline $\mathrm{rs} 1005230^{\mathrm{a}}$ & $-1451 \mathrm{~T} \rightarrow \mathrm{C}$ & & & & \\
\hline rs35864111 ${ }^{a}$ & $-14114 \mathrm{G} \rightarrow 5 \mathrm{G}$ & & & & \\
\hline $\begin{array}{l}\text { rs833061 } \\
\text { rs13207351 }\end{array}$ & $\begin{array}{l}-460 \mathrm{~T} \rightarrow \mathrm{C} \\
-152 \mathrm{G} \rightarrow \mathrm{A}\end{array}$ & \multirow[t]{2}{*}{$\begin{array}{l}\text { F5'-ACA GGG AAG CTG GGT GAA TGG AGC G-3' } \\
\text { R5'-CGG GGA CAG GCG AGC CTC AG-3' }\end{array}$} & \multirow[t]{2}{*}{ Promoter } & \multirow[t]{2}{*}{464} & \multirow[t]{2}{*}{65} \\
\hline rs 1570360 & $-1154 \mathrm{G} \rightarrow \mathrm{A}^{\mathrm{c}}$ & & & & \\
\hline rs2010963 & $-634 \mathrm{G} \rightarrow \mathrm{C}^{\mathrm{d}}$ & $\begin{array}{l}\text { F5'-CGA CGG CTT GGG GAG ATT GC-3' R5'-GGC } \\
\text { GGT CAC CCC CAA AAG C-3' }\end{array}$ & $5^{\prime} \mathrm{UTR}$ & 194 & 60 \\
\hline
\end{tabular}

\footnotetext{
${ }^{\text {a }}$ Polymorphisms in complete linkage disequilibrium

${ }^{\mathrm{b}}$ Reported also in the literature as $-1540 \mathrm{~A} / \mathrm{C}$

${ }^{\mathrm{c}}$ Reported also in the literature as $-116 \mathrm{G} / \mathrm{A}$

${ }^{\mathrm{d}}$ Reported also in the literature as $+405 \mathrm{G} / \mathrm{C}$
} 
progression or death from any cause. If a patient had not progressed or died, PFS was censored at the time of the last follow-up.

The secondary endpoint was association between VGPs and (1) overall RECIST-defined radiological response rate (ORR) divided into two main categories: presence (complete + partial responses) or absence (stable + progression of disease) of radiological tumor response, (2) OS, defined as time from study entry to death from all causes.

The association between each gene polymorphism and ORR was evaluated by Fisher's exact test. The association between polymorphisms and PFS and OS was examined using Kaplan-Meier curves and log-rank test.

In the univariate survival analysis, the estimated relative risk and its associated $95 \%$ confidence interval $(95 \% \mathrm{CI})$ were based on the log-rank test.

Cox proportional hazards regression model was used to evaluate the association between VGPs along with biochemical covariates known to have prognostic value in MCRC $[20,21]$ and the primary endpoint (PFS) in the multivariate analysis. The enter method was used to enter independent variables into the model (i.e., all variables were entered into the model in one single step without checking for significance).

All statistical tests were two-sided. Analyses were done using MedCalc for Windows, version 9.5.0.0 (MedCalc Software, Mariakerke, Belgium).

Lewontin's standardized disequilibrium coefficient $\left(D^{\prime}\right)$ among investigated VGPs was assessed using the Haploview program (Barrett Bioinformatics 2005). This software provides the disequilibrium coefficient $D^{\prime}$ as a measure of the nonrandom association of alleles at different loci. $D^{\prime}$ coefficient is equal to 1 only if two SNPs have not been separated by recombination (or recurrent mutation) during the history of the sample (complete degree of linkage disequilibrium (LD)).

In our study, VGPs $-2578,-1512,-1451,-1411$, and -460 were in complete LD and therefore they were analyzed as haplotype (Haplo1 and Haplo2) for association to clinical outcome (Haplo1 for the A-18 bp insertion-T-4 G-C combination and Haplo2 for the C18 bp deletion-C-5 G-T combination).

\section{Results}

Patients' characteristics and treatment delivery

Between January 2006 and August 2008, 40 patients were entered into the study. Enrolment was closed in August 2008 to guarantee an adequate follow-up.

All included patients were treated with FOLFIRI+ bevacizumab as per protocol for at least two cycles.
The median age at study entry was 61 years (range, 30-78). Thirty-eight patients were Caucasian, one African/American, and one Hispanic.

ECOG PS was 0 for 34 patients and 1 for six patients. Detailed demographic and clinical characteristics of study patients are summarized in Table 2.

Median number of treatment cycles administered was 7 (range, 3-19). Dose reduction ( $>25 \%$ of the planned dose for any of the drug for two or more cycles) or treatment delay ( $>1$ week of delay for two or more cycles) was necessary in eight patients $(20 \%)$. In Table 2 are also reported genotypes and allele/haplotype frequencies of investigated VGPs.

\section{Clinical outcome}

\section{Progression-free survival}

Twenty-seven of 40 patients reached the PFS endpoint with a median follow-up for progression-free patients of 15 months.

The median PFS (mPFS) for the whole cohort was 10.8 months (95\% CI, 5.9-19.2).

PFS was analyzed according to VGPs genotypes. The 17 patients homozygous for the VEGF $-2578,-1512,-1451$, $-1411,-460$ C-18 bp deletion-C-5 G-T haplotype (Haplo2) had significantly shorter mPFS compared to the other 23 patients, i.e., three patients homozygous for the A-18 bp insertion-T-4 G-C haplotype (Haplo1) +20 heterozygous patients (Haplo1/Haplo2), with a mPFS of 9 months and 15.4 months, respectively ( $p=0.02$, hazard ratio (HR) 2.64 , $95 \% \mathrm{CI}=1.13-6.16$; Figure 1a).

Also, the VGPs $-152(\mathrm{G} / \mathrm{G}$ vs. G/A+A/A) and -1154 $(\mathrm{G} / \mathrm{G}$ vs. $\mathrm{G} / \mathrm{A}+\mathrm{A} / \mathrm{A})$ were significantly associated with PFS, with a mPFS of 8.9 vs. 15.4 months $(p=0.007$; $\mathrm{HR}, 3.53$; $95 \% \mathrm{CI}, 1.40-8.86)$ and 9.8 vs. 16 months $(p=$ 0.03 ; HR, 2.32; 95\%CI, 1.08-5.01), respectively (Fig. 1b, c).

The VGP -634 did not significantly affect PFS with a mPFS of 13.6, 14, and 9.3 months for $\mathrm{C} / \mathrm{C}, \mathrm{C} / \mathrm{G}$, and $\mathrm{G} / \mathrm{G}$ genotypes, respectively, $p=0.83$. (Figure $1 \mathrm{~d}$ ).

VGPs found to be significantly associated with PFS in the Kaplan-Meier analysis (Fig. 1) were evaluated in a multivariate Cox regression analysis for PFS along with other biochemical covariates (i.e., white blood cells, lactate dehydrogenase, alkaline phosphatase, and CA19.9) previously documented as having prognostic properties in MCRC patients treated with first-line chemotherapy $[20,21]$. Only the VGP -1154 was found to be independently associated with PFS with the GG genotype conferring a nearly fourfold higher risk of early progression compared to $\mathrm{GA}+\mathrm{AA}$, HR ,4.43; $95 \% \mathrm{CI}$, $1.28-15.36 ; p=0.02$ (Table 3 ). 
Table 2 Characteristics of the 40 study patients

\begin{tabular}{|c|c|c|c|c|}
\hline Sex & & $\mathrm{LDH}$ & & \\
\hline Male & 22 & & Median & $301 \mathrm{U} / 1$ \\
\hline Female & 18 & & Range & $63-724 \mathrm{U} / 1$ \\
\hline Age & & ALP & & \\
\hline Median & 61 years & & Median & $13.1 \mathrm{U} / 1$ \\
\hline Range & $30-78$ years & & Range & $7.8-16.2 \mathrm{U} / 1$ \\
\hline Race & & ECOG PS & & \\
\hline Caucasian & 38 & & 0 & 34 \\
\hline African/American & 1 & & 1 & 6 \\
\hline Hispanic & 1 & Mets & & \\
\hline Primary & & & Other than liver & 8 \\
\hline Colon & 33 & & Only liver & 14 \\
\hline Rectum & 7 & & Liver+other sites & 18 \\
\hline Histologic subtype & & VGPs & & \\
\hline Mucinous & 12 & Five VGPs in complete $\mathrm{LD}^{\mathrm{a}}$ & Haplo1/haplo1 & 3 \\
\hline Non-mucinous & 28 & & Haplo1/Haplo2 & 20 \\
\hline Histologic grading & & & Haplo2/Haplo2 & 17 \\
\hline Grade $1-2$ & 37 & -152 & $\mathrm{~A} / \mathrm{A}$ & 4 \\
\hline Grade 3 & 3 & & $\mathrm{~A} / \mathrm{G}$ & 21 \\
\hline No. of treatment cycles & & & $\mathrm{G} / \mathrm{G}$ & 15 \\
\hline Median & Seven cycles & -1154 & $\mathrm{~A} / \mathrm{A}$ & 4 \\
\hline Range & 3-19 cycles & & $\mathrm{A} / \mathrm{G}$ & 11 \\
\hline Baseline CEA & & & $\mathrm{G} / \mathrm{G}$ & 25 \\
\hline Median & $3.5 \mathrm{ng} / \mathrm{ml}$ & -634 & $\mathrm{C} / \mathrm{C}$ & 7 \\
\hline Range & $0.7-218.7 \mathrm{ng} / \mathrm{ml}$ & & $\mathrm{C} / \mathrm{G}$ & 26 \\
\hline Baseline CA19.9 & & & $\mathrm{G} / \mathrm{G}$ & 7 \\
\hline Median & $9.9 \mathrm{U} / \mathrm{ml}$ & Minor allele frequency & Haplo1 & $32.5 \%$ \\
\hline Range & $0.6-362.2 \mathrm{U} / \mathrm{ml}$ & & $-152 * A$ & $36.2 \%$ \\
\hline WBC & & & $-1154 * \mathrm{~A}$ & $23.7 \%$ \\
\hline Median & $7.3^{*} 10^{3} / \mathrm{mm}^{3}$ & & $-634 * \mathrm{C}$ & $50 \%$ \\
\hline Range & $2.3-17.0 * 10^{3} / \mathrm{mm}^{3}$ & & & \\
\hline
\end{tabular}

Values refer to absolute number of patients except when otherwise specified

$N$ number, $W B C$ white blood cells, $L D H$ lactate dehydrogenase, $A L P$ alkaline phosphatase, ECOG PS Eastern Cooperative Oncology Group performance status, VGPS VEGF gene polymorphisms, $L D$ linkage disequilibrium

${ }^{a}$ In our study, VGPs $-2578,-1512,-1451,-1411$, and -460 were in complete LD, and therefore, they were analyzed as haplotype (Haplo1 and Haplo2) for association to clinical outcome (Haplo1 for the A-18 bp insertion-T-4 G-C combination and Haplo2 for the C-18 bp deletion-C-5 G-T combination)

\section{Overall survival}

Only 15 deaths (38\%) out of 40 study subjects occurred after a median follow-up for surviving patients of 18.4 months (range, 3.7-36.6). No significant influence on OS has been observed for the investigated polymorphisms (data not shown); however, more death events are necessary before drawing definitive conclusions in this respect. The mOS for the entire cohort was 20.6 months $(95 \% \mathrm{CI}, 11.1-32.2)$.

\section{Overall radiologic response rate}

Frequencies of alleles and genotypes were analyzed according to treatment response (Table 4).
Among all the VGPs, only VGP -634 was significantly associated with ORR, with nearly a threefold increase in the chance of response for patients carrying the $\mathrm{G} / \mathrm{G}$ variant: $\mathrm{ORR}$ for $\mathrm{G} / \mathrm{G}$ vs. $\mathrm{G} / \mathrm{C}+\mathrm{C} / \mathrm{C}=64 \%$ vs. $14 \%$, Fisher's test $p=0.03$.

\section{Toxicity}

In Table 5 are reported frequencies of grade $3 / 4$ adverse events. Results are comparable to previously reported data on FOLFIRI+bevacizumab regimen [27]. No significant differences in toxicity were observed across the different VEGF genotypes (data not shown); however, sample size and grade $3 / 4$ adverse event frequencies were too modest to draw definitive conclusions. 
a

VGPS $-2578,-1512,-1451,-1411$ and -460

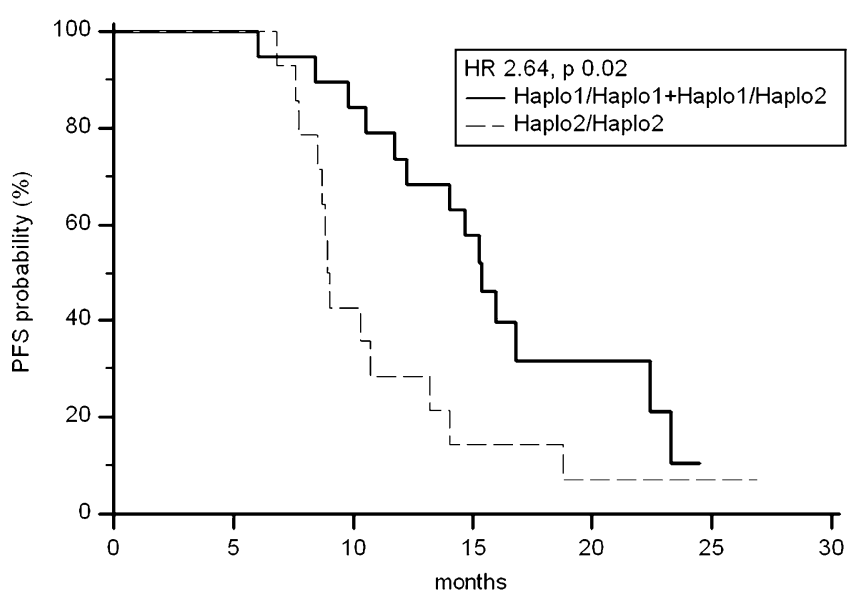

C



Fig. 1 VEGF gene polymorphisms (VGP) and progression-free survival (PFS). a PFS according to the $-2578,-1512,-1451,-1411$, and -460 VGP genotypes. These five VGPs were in complete linkage disequilibrium, being the haplotype-1 (Haplo1) the A-18 bp insertion-T4G-C combination and the haplotype-2 (Haplo2) the C-18 bp deletionC-5G-T combination. mPFS was 9 months for the Haplo2/Haplo2 and 15.4 months for the Haplo1/Haplo2 or Haplo1/Haplo1 genotype. b PFS
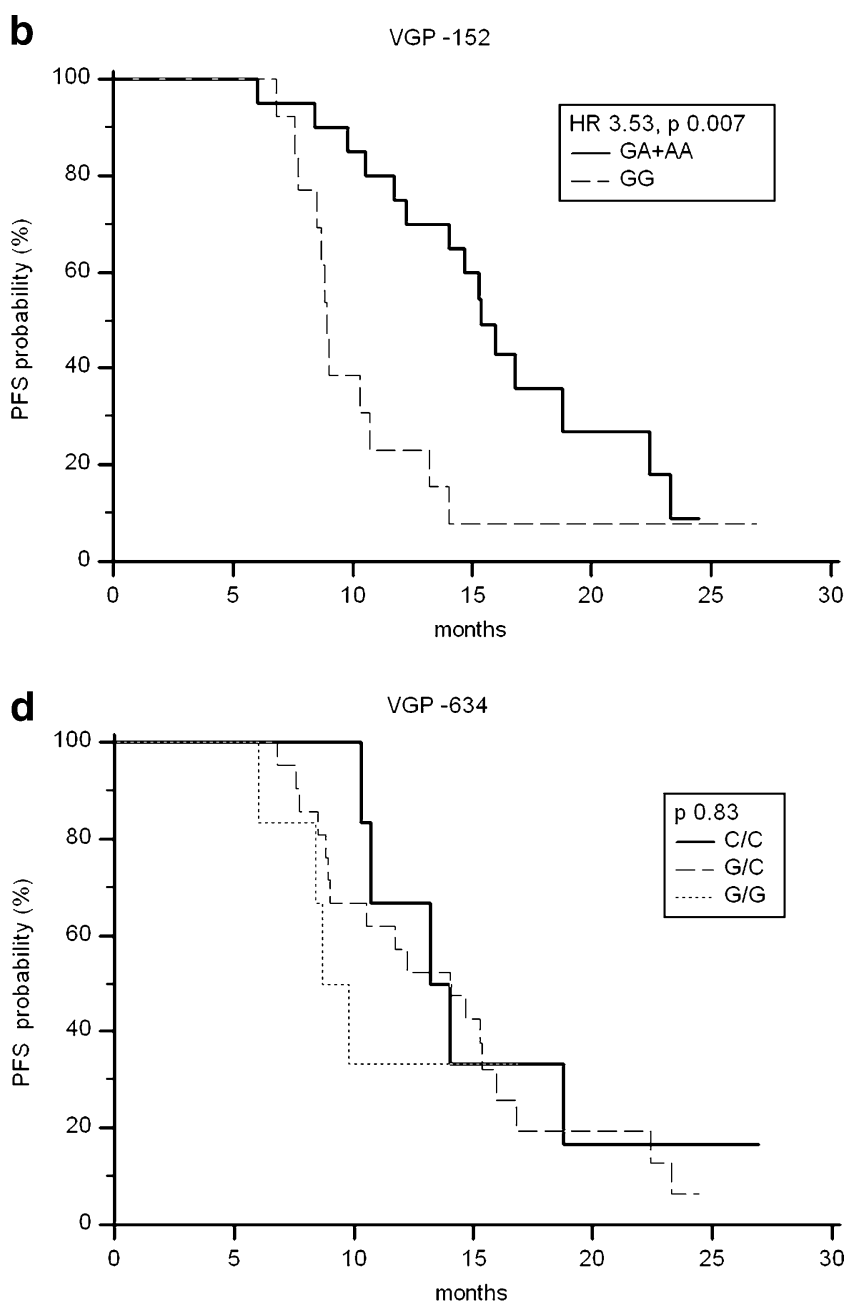

according to the -152 VGP genotypes. mPFS was 8.9 months for the $\mathrm{G} / \mathrm{G}$ and 15.4 months for the G/A or A/A genotype. c PFS according to the -1154 VGP genotypes. mPFS was 9.8 months for the $\mathrm{G} / \mathrm{G}$ and 16.0 months for the G/A or A/A genotype. d PFS according to the -634 VGP genotypes. mPFS was 13.6 months for the $\mathrm{C} / \mathrm{C}, 14.0$ months for the $\mathrm{C} / \mathrm{G}$, and 9.3 months for the $\mathrm{G} / \mathrm{G}$ genotype; $H R$ hazard ratio

Table 3 Cox regression multivariate analysis for progression-free survival (PFS)

\begin{tabular}{|c|c|c|c|}
\hline Covariate & HR & $95 \% \mathrm{CI}$ of $\mathrm{HR}$ & $p$ \\
\hline ALP & 0.992 & 0.979 to 1.006 & 0.266 \\
\hline CA 19.9 & 1.006 & 0.999 to 1.012 & 0.082 \\
\hline LDH & 1.003 & 0.999 to 1.006 & 0.111 \\
\hline WBC & 0.933 & 0.804 to 1.082 & 0.361 \\
\hline Five VGPs in complete LD ${ }^{\mathrm{a}}$ (Haplo1Haplo1+Haplo1Haplo2/Haplo2Haplo2) & 0.290 & 0.030 to 2.807 & 0.288 \\
\hline VEGF $-152(\mathrm{GG} / \mathrm{GA}+\mathrm{AA})$ & 3.780 & 0.427 to 33.466 & 0.234 \\
\hline VEGF -1154 (GG/GA+AA) & 4.426 & 1.276 to 15.359 & 0.02 \\
\hline
\end{tabular}

ALP, CA19.9, LDH, and WBC were analyzed as continuous variables

$A L P$ alkaline phosphatase, $L D H$ lactate dehydrogenase, $W B C$ white blood cells, $H R$ hazard ratio, $C I$ confidence interval, VGPS VEGF gene polymorphisms, $L D$ linkage disequilibrium

${ }^{\text {a }}$ In our study, VGPs $-2578,-1512,-1451,-1411$, and -460 were in complete LD, and therefore, they were analyzed as haplotype (Haplo1 and Haplo2) for association to clinical outcome (Haplo1 for the A-18 bp insertion-T-4 G-C combination and Haplo2 for the C-18 bp deletion-C-5 G-T combination) 
Table 4 Polymorphisms and response rate

\begin{tabular}{|c|c|c|c|c|}
\hline VGP & Genetic Variants & ORR & Odds Ratio $(95 \% \mathrm{CI})$ & Fisher's $p$ value \\
\hline \multirow[t]{2}{*}{ VGPs in complete $\mathrm{LD}^{\mathrm{a}}$} & Haplo1/Haplo1 + Haplo1/Haplo2 & $57 \%$ & $1.16(0.33-4.07)$ & p 0.82 \\
\hline & Haplo2/Haplo2 & $53 \%$ & & \\
\hline \multirow[t]{2}{*}{ VEGF -152} & $\mathrm{G} / \mathrm{A}+\mathrm{A} / \mathrm{A}$ & $60 \%$ & $1.71(0.47-6.24)$ & p 0.14 \\
\hline & $\mathrm{G} / \mathrm{G}$ & $37 \%$ & & \\
\hline \multirow[t]{2}{*}{ VEGF -1154 } & $\mathrm{A} / \mathrm{A}+\mathrm{A} / \mathrm{G}$ & $53 \%$ & $0.90(0.25-3.25)$ & p 0.87 \\
\hline & $\mathrm{G} / \mathrm{G}$ & $56 \%$ & & \\
\hline \multirow[t]{2}{*}{ VEGF -634} & $\mathrm{C} / \mathrm{C}+\mathrm{C} / \mathrm{G}$ & $64 \%$ & $10.50(1.13-97.91)$ & p 0.03 \\
\hline & $\mathrm{G} / \mathrm{G}$ & $14 \%$ & & \\
\hline
\end{tabular}

$V G P$ VEGF gene polymorphisms, ORR overall radiologic response rate, $C I$ confidence interval, $L D$ linkage disequilibrium

${ }^{a}$ In our study, VGPs $-2578,-1512,-1451,-1411$, and -460 were in complete LD, and therefore, they were analyzed as haplotype (Haplo1 and Haplo2) for association to clinical outcome (Haplo1 for the A-18 bp insertion-T-4 G-C combination and Haplo2 for the C-18 bp deletion-C-5 G-T combination)

\section{Discussion}

The purpose of this study was to evaluate the impact of germline VGPs located in the promoter/5, UTR on the clinical outcome of MCRC patients treated with the antiVEGF antibody bevacizumab.

The hypothesis was that VGPs may influence bevacizumab efficacy by interfering with the expression of its molecular target, i.e., extracellular VEGF.

VEGF is a key mediator of tumor neo-angiogenesis and promotes tumor growth and metastases. During the cancer process, expression of VEGF is induced in both normal and cancer cells.

VEGF originating from normal myeloid cells in response to tumor stimulation may be essential in tumorigenesis and may reduce standard chemotherapy activity [7]. During cancer progression, angiogenesis (as a host-driven process) is

Table 5 Common grade 3/4 adverse events

\begin{tabular}{lcc}
\hline Adverse event grade $3 / 4$ & $N$ & $\%$ \\
\hline Neutropenia & 14 & 35 \\
Leucopenia & 8 & 20 \\
Diarrhea & 5 & 12.5 \\
Febrile neutropenia & 4 & 10 \\
DVT & 3 & 7.5 \\
Hypertension & 2 & 5 \\
Mucositis & 2 & 5 \\
Thrombocytopenia & 1 & 2.5 \\
Anemia & 1 & 2.5 \\
Nausea/vomiting & 1 & 2.5 \\
Bleeding & 1 & 2.5 \\
Fistula/perforation & 1 & 2.5 \\
\hline
\end{tabular}

$D V T$ deep vein thrombosis, $N$ number of patients, $\%$ percentage of patients within the group likely to be influenced by germline genetic variability more than tumor genetic variability. In a previous study by Schneider et al. [15], detection of VEGF -2578 AA and -1154 AA+ AG genotypes in tumor genomic DNA was associated with improved overall survival in breast cancer patients treated with bevacizumab+paclitaxel. However, the same authors considered the VGPs analysis performed on tumor DNA and the lack of available peripheral blood samples as limitations of the study. According to Schneider et al., clinically meaningful polymorphisms of angiogenesis genes should be found, in fact, in normal germline DNA because tumor angiogenesis is mediated by "normal" endothelial, hematopoietic, and stromal cells [22].

We chose to investigate germline VGPs in the promoter region as crucial regulation point. In fact, host VEGF promoter activity has been demonstrated to be induced by tumor cells around and throughout the tumor mass as expression of activation in normal fibroblast and myeloid cells [28].

Moreover, several functional polymorphisms in the VEGF promoter/5'UTR have been described. In vitro studies have demonstrated that the VEGF $-2578 \mathrm{CC}$ and the -1154 GG genotypes are associated with an increased VEGF secretion in human blood mononuclear cells [23, 29, 30].

As a confirmation of preclinical findings, genotypes determining a higher VEGF secretion have been found in humans to be associated with increased risk of prostate, bladder, lung, colorectal, ovarian, and breast cancer diagnosis or relapse [9-14].

In our study, we found a statistically significant association between germline $-2578,-1512,-1451$, $-1411,-460,-152$, and -1154 VGPs and PFS. Moreover, by analyzing VGPs along with biochemical covariates known to influence prognosis in MCRC patients in a multivariate Cox regression analysis, VGP -1154 was the only covariate independently associated with PFS, with the 
GG genotype conferring a nearly fourfold higher risk of early progression compared to $\mathrm{GA}+\mathrm{AA}, \mathrm{HR}, 4.43 ; 95 \% \mathrm{CI}$, 1.28-15.36; $p=0.02$ (Table 3).

Hence, VEGF hypersecretion may account for the significant and independent worse outcome seen in our study in bevacizumab-treated patients carrying the VEGF -1154 GG genotype.

Only recently, the influence of VGPs on the efficacy of the anti-VEGF monoclonal antibody bevacizumab has been investigated in breast and ovarian cancer $[15,16]$, and the present study is, to the best of our knowledge, the first report demonstrating a predictive value for PFS of VGPs in bevacizumab-treated MCRC patients.

Even if not completely comparable, our results are in keeping with Schneider's findings in breast cancer [15]. In the Schneider study in fact genotype AA in both -2578 and -1154 VEGF loci were associated with a longer OS of bevacizumab-treated patients (HR, $0.58 ; P=0.023$ and HR, $0.62 ; P=.001$, respectively). Similarly, in our cohort, AA genotype in those loci was associated with a significantly longer PFS as compared to the alternate genotypes (see Table 3 and Fig. 1).

We also found a significant association of germline VGP -634 with ORR, but not with PFS (Table 4).

It is not unusual for cancer patients that an enhanced radiologic response does not translate in a longer PFS [27, 31]. Two alternative hypotheses might be generated to explain this finding: either (1) a higher number of subjects are required to detect a significant difference in PFS for this group of patients, or (2) VEGF $-634 \mathrm{C} / \mathrm{C}+\mathrm{C} / \mathrm{G}$ genotype is associated to a tumor which is more chemosensitive (which would explain the higher response rate) and at the same time more aggressive (which would explain why a gain in ORR did not translate in a gain in PFS). Confirmation of this finding is needed in future studies.

The identification of predictive markers of bevacizumab efficacy is a fascinating area of research [21,32] and may lead to a further tailoring of anticancer treatments thus optimizing their efficacy. As an example, a high dose trial of bevacizumab might be designed to improve treatment efficacy in subjects genetically predisposed to overexpress VEGF.

A prospective trial on a larger population comparing chemotherapy+bevacizumab with chemotherapy only is required to definitively determine how much VGPs are predictive (i.e., impacting on treatment efficacy) rather than prognostic (i.e., impacting on overall survival and cancer natural history) in this cancer population.

We acknowledge that the sample size of the present study is relatively small. However, we found difference in PFS or RR between genotype groups to be statistically significant ( $p$ value ranging from 0.03 to 0.07 ), leading to the hypothesis that the effect of VEGF genetic variations on clinical outcomes might be very large in magnitude, and a limited number of patients may be sufficient to detect statistically meaningful differences. We are currently running a phase III trial of bevacizumab in metastatic colorectal cancer patients where VGPs are prospectively analyzed and their association with clinical outcomes has been pre-planned in the protocol as secondary endpoint, but data are not mature enough at the moment to further increase our patient sample size. Nonetheless, the statistically significant results obtained from these 40 patients together with the novelty of the findings might serve now as a base for future researches of the scientific community.

Gene polymorphism analysis represents in fact a more "reproducible" and easier test to perform compared to the measurement of soluble mediators such as plasma VEGF and, although larger prospective clinical trials are needed, according to our results, VGPs analysis has the potential for discriminating patients who would most benefit from bevacizumab in MCRC patients at standard dose.

Acknowledgments The authors wish to thank Barbara Leone for her excellent technical assistance. This study was partially supported by the Italian Ministry of Health Research Grant RFPS-2006-7-342220 and ACC-WP 3/1b and carried out within the Experimental Physiopathology-Oncology Rehabilitation $\mathrm{PhD}$ program, 'Tor Vergata' University of Rome.

Conflict of interest statement The authors declare that they have no conflict of interest in connection with this paper.

\section{References}

1. Jemal A, Siegel R, Ward E, Hao Y, Xu J, Thun MJ (2009) Cancer statistics, 2009. CA Cancer J Clin 59:225-249

2. Köhne CH, Lenz HJ (2009) Chemotherapy with targeted agents for the treatment of metastatic colorectal cancer. Oncologist $14: 478-488$

3. Heath VL, Bicknell R (2009) Anticancer strategies involving the vasculature. Nat Rev Clin Oncol 6:395-404

4. Karapetis CS, Khambata-Ford S, Jonker DJ, O'Callaghan CJ, Tu $\mathrm{D}$, Tebbutt NC et al (2008) K-ras mutations and benefit from cetuximab in advanced colorectal cancer. $\mathrm{N}$ Engl J Med 359:1757-1765

5. Loupakis F, Pollina L, Stasi I, Ruzzo A, Scartozzi M, Santini D et al (2009) PTEN expression and KRAS mutations on primary tumors and metastases in the prediction of benefit from cetuximab plus irinotecan for patients with metastatic colorectal cancer. J Clin Oncol 27:2622-2629

6. Ince WL, Jubb AM, Holden SN, Holmgren EB, Tobin P, Sridhar $\mathrm{M}$ et al (2005) Association of k-ras, b-raf, and p53 status with the treatment effect of bevacizumab. J Natl Cancer Inst 97:981-989

7. Stockmann C, Doedens A, Weidemann A, Zhang N, Takeda N, Greenberg JI et al (2008) Deletion of vascular endothelial growth factor in myeloid cells accelerates tumorigenesis. Nature 456:814-818

8. Stevens A, Soden J, Brenchley PE, Ralph S, Ray DW (2003) Haplotype analysis of the polymorphic human vascular endothelial growth factor gene promoter. Cancer Res 63:812-816 
9. McCarron SL, Edwards S, Evans PR, Gibbs R, Dearnaley DP, Dowe A et al (2002) Influence of cytokine gene polymorphisms on the development of prostate cancer. Cancer Res 62:3369-3372

10. García-Closas M, Malats N, Real FX, Yeager M, Welch R, Silverman D et al (2007) Large-scale evaluation of candidate genes identifies associations between VEGF polymorphisms and bladder cancer risk. PLoS Genet 3:e29

11. Heist RS, Zhai R, Liu G, Zhou W, Lin X, Su L, Asomaning K et al (2008) VEGF polymorphisms and survival in early-stage nonsmall-cell lung cancer. J Clin Oncol 26:856-862

12. Kim JG, Chae YS, Sohn SK, Cho YY, Moon JH, Park JY et al (2008) Vascular endothelial growth factor gene polymorphisms associated with prognosis for patients with colorectal cancer. Clin Cancer Res 14:62-66

13. Hefler LA, Mustea A, Könsgen D, Concin N, Tanner B, Strick R et al (2007) Vascular endothelial growth factor gene polymorphisms are associated with prognosis in ovarian cancer. Clin Cancer Res 13:898-901

14. Jin Q, Hemminki K, Enquist K, Lenner P, Grzybowska E, Klaes R et al (2005) Vascular endothelial growth factor polymorphisms in relation to breast cancer development and prognosis. Clin Cancer Res 11:3647-3653

15. Schneider BP, Wang M, Radovich M, Sledge GW, Badve S, Thor A et al (2008) Association of vascular endothelial growth factor and vascular endothelial growth factor receptor-2 genetic polymorphisms with outcome in a trial of paclitaxel compared with paclitaxel plus bevacizumab in advanced breast cancer: ECOG 2100. J Clin Oncol 26:4672-4678

16. Schultheis AM, Lurje G, Rhodes KE, Zhang W, Yang D, Garcia AA et al (2008) Polymorphisms and clinical outcome in recurrent ovarian cancer treated with cyclophosphamide and bevacizumab. Clin Cancer Res 14:7554-7563

17. Saltz LB, Clarke S, Díaz-Rubio E, Scheithauer W, Figer A, Wong $R$ et al (2008) Bevacizumab in combination with oxaliplatin-based chemotherapy as first-line therapy in metastatic colorectal cancer: a randomized phase III study. J Clin Oncol 26:2013-2019

18. Fuchs CS, Marshall J, Mitchell E, Wierzbicki R, Ganju V, Jeffery $M$ et al (2007) Randomized, controlled trial of irinotecan plus infusional, bolus, or oral fluoropyrimidines in first-line treatment of metastatic colorectal cancer: results from the BICC-C Study. J Clin Oncol 25:4779-4786

19. Therasse P, Arbuck SG, Eisenhauer EA, Wanders J, Kaplan RS, Rubinstein L et al (2000) New guidelines to evaluate the response to treatment in solid tumors. European Organization for Research and Treatment of Cancer, National Cancer Institute of the United States, National Cancer Institute of Canada. J Natl Cancer Inst 92:205-216

20. Köhne CH, Cunningham D, Di CF, Glimelius B, Blijham G, Aranda $E$ et al (2002) Clinical determinants of survival in patients with 5-fluorouracil-based treatment for metastatic colorectal cancer: results of a multivariate analysis of 3,825 patients. Ann Oncol 13:308-317

21. Formica V, Massara MC, Portarena I, Fiaschetti V, Grenga I, Del Vecchio BG et al (2009) Role of CA19.9 in predicting bevacizumab efficacy for metastatic colorectal cancer patients. Cancer Biomark 5:167-175

22. Schneider BP, Radovich M, Miller KD (2009) The role of vascular endothelial growth factor genetic variability in cancer. Clin Cancer Res 15:5297-5302

23. Shahbazi M, Fryer AA, Pravica V, Brogan IJ, Ramsay HM, Hutchinson IV et al (2002) Vascular endothelial growth factor gene polymorphisms are associated with acute renal allograft rejection. J Am Soc Nephrol 13:260-264

24. Watson CJ, Webb NJ, Bottomley MJ, Brenchley PE (2000) Identification of polymorphisms within the vascular endothelial growth factor (VEGF) gene: correlation with variation in VEGF protein production. Cytokine 12:1232-1235

25. Chen Y, Cicciarelli J, Pravica V, Hutchinson IV (2009) Longrange linkage on chromosome $6 p$ of VEGF, FKBP5, HLA and TNF alleles associated with transplant rejection. Mol Immunol 47:96-100

26. Davis AH, Wang J, Tsang TC, Harris DT (2007) Direct sequencing is more accurate and feasible in detecting single nucleotide polymorphisms than RFLP: using human vascular endothelial growth factor gene as a model. Biol Res Nurs 9:170-178

27. Maisey N, Chau I, Cunningham D, Norman A, Seymour M, Hickish $\mathrm{T}$ et al (2002) Multicenter randomized phase III trial comparing protracted venous infusion (PVI) fluorouracil (5-FU) with PVI 5-FU plus mitomycin in inoperable pancreatic cancer. J Clin Oncol 20:3130-3136

28. Fukumura D, Xavier R, Sugiura T, Chen Y, Park EC, Lu N et al (1998) Tumor induction of VEGF promoter activity in stromal cells. Cell 94:715-725

29. Mohammadi M, Ollier W, Hutchinson I (2003) A functional association study of VEGF gene polymorphisms with VEGF expression by stimulated PBM cells. Hum Immunol 64:S125

30. Awata T, Inoue K, Kurihara S, Ohkubo T, Watanabe M, Inukai K et al (2002) A common polymorphism in the 5 '-untranslated region of the VEGF gene is associated with diabetic retinopathy in type 2 diabetes. Diabetes 51:1635-1639

31. Cocconi G, Bella M, Zironi S, Algeri R, Di Costanzo F, De Lisi V et al (1994) Fluorouracil, doxorubicin, and mitomycin combination versus PELF chemotherapy in advanced gastric cancer: a prospective randomized trial of the Italian Oncology Group for Clinical Research. J Clin Oncol 12:2687-2693

32. Jubb AM, Hurwitz HI, Bai W, Holmgren EB, Tobin P, Guerrero AS et al (2006) Impact of vascular endothelial growth factor-A expression, thrombospondin-2 expression, and microvessel density on the treatment effect of bevacizumab in metastatic colorectal cancer. J Clin Oncol 24:217-227 